\title{
Domain walls, domain wall transformations and structural changes in permalloy nanowires when subjected to current pulses
}

\author{
E.-M. Hempe ${ }^{1,2}$, M. Kläuï ${ }^{*}$, T. Kasama ${ }^{1}$, D. Backes ${ }^{3,4}$, F. Junginger ${ }^{1,3}$, S. Krzyk ${ }^{3}$, \\ L. J. Heyderman ${ }^{4}$, R. Dunin-Borkowski**, ${ }^{*}$, and U. Rüdiger ${ }^{3}$ \\ 'Department of Materials Science and Metallurgy, University of Cambridge, Pembroke Street, \\ Cambridge CB2 3QZ, United Kingdom \\ 2 Department of Physics, Universität Regensburg, Universitätsstraße 31, 93040 Regensburg, Germany \\ ${ }^{3}$ Fachbereich Physik, Universität Konstanz, Universitätsstraße 10, 78457 Konstanz, Germany \\ ${ }^{4}$ Laboratory for Micro- and Nanotechnology, Paul Scherrer Institut, 5232 Villigen PSI, Switzerland
}

\begin{abstract}
PACS 68.37.Lp, 75.60.Ch, 75.75.+a
We report the direct transmission electron microscopy observation of spin structure transformations in nanoscale Permalloy zigzag wires due to Joule heating during the injection of current pulses. This heating is sufficient to overcome the energy barriers separating the different metastable domain wall spin structures. Due to the large energy barriers these are stable and observable at room temperature by off-axis electron holography and Fresnel imaging. The interaction between different domain walls is probed and the main pinning mechanism is determined to be the edge roughness. In addition to transformations, we also report on thermally assisted domain wall hopping between two pinning sites and structural changes that occur when the samples are subjected to even higher current pulses.
\end{abstract}

Domain walls and magnetization reversal by domain wall motion are currently at the center of interest as they allow for the investigation of fundamental physical questions, e.g. how the geometry influences the spin structure [1], why and how domain walls are pinned at constrictions [2] as well as the details of the domain wall propagation process in confined structures [3]. First applications have been proposed for data storage [4] and logic [5]. The recent work on current-induced domain wall motion (CIDM) [3, 6-9] opens up the possibility to manipulate the magnetic state of the sample by currents instead of external fields, allowing simpler device design avoiding field-generating strip lines. While control of domain walls in experiments is quite good, the theory of the underlying process is still disputed [10-15].

Since the necessary current densities are quite large, there are, besides CIDM, also effects caused by Joule heating, which can be substantial in certain geometries. It has been predicted and subsequently shown that domain wall transformations not only stem from spin transfer torque $[8,16-18]$ but can also be caused by thermal excitations [19]. Because the random nature of thermal effects is detrimental to the proposed applications, these effects have to be distinguished from the desirable spin torque effects and if possible suppressed by suitable cooling mechanisms [20]. However, these effects also open up the possibility to learn more about the spin structure of domain walls, their interactions and potential landscape, which will be investigated here.

\footnotetext{
"Corresponding author: e-mail: mathias.klaeui@uni-konstanz.de

** Center for Electron Nanoscopy, DTU, 2800 Denmark
} 
In order to observe the response of the domain walls and their detailed spin structure to currents, high spatial resolution imaging of the nanoscale domain wall spin structure is necessary. Because of the very high obtainable spatial resolution $(<10 \mathrm{~nm})$ [2], transmission electron microscopy is especially apt for such measurements. Inherent in its nature as a transmission technique is the requirement that the samples have to be patterned on very thin (e.g. $\mathrm{Si}_{3} \mathrm{~N}_{4}$ ) membranes [21, 22], which are poor heat conductors. Thus the Joule heat caused by the currents is ineffectively dissipated, which leads to a significant temperature rise. We used, unless noted otherwise, $10^{-5} \mathrm{~s}$ long rectangular current pulses because the wires cannot sustain the necessary current densities for longer periods of time without incurring structural demage.

Samples consisting of four Permalloy $\left(\mathrm{Ni}_{80} \mathrm{Fe}_{20}\right)$ zigzag wires (widths: $240-560 \mathrm{~nm}$, thicknesses: 12-34 nm), two with one kink and two with three kinks, were fabricated [22]. The straight wire segments which make up this zigzag structure have a length of $30 \mu \mathrm{m}$ and $14 \mu \mathrm{m}$, respectively. Due to the zigzag geometry, domain walls can be easily and reproducibly initialized by applying a strong in-plane magnetic field perpendicular to the average wire axis. When the field is reduced back to zero the magnetization will align with the nearest direction parallel to the wire axis in order to minimize the stray field energy and thus lead to alternating head-to-head and tail-to-tail domain walls in the kinks.

In order to observe the domain walls, we used off-axis electron holography [23] and Fresnel imaging [24]. While the first technique, which requires time-consuming data processing, reveals detailed information about the spin structure, the latter one is much easier but yields only indirect information; the black and white contrast observed originates from the electron beam which is deflected due to the Lorentz force [25]. The contrast of the vortices is characteristic for the direction of the vortex circulation (chirality).

So far the head-to-head domain wall spin structures that have raised most interest are the transverse and vortex walls $[19,26,27]$. But as already shown previously, other domain wall types also exist [18] but the details of the energetics and the spin structures have previously been neglected.

In the first set of wires (set 1), the thinnest and narrowest ones ( $240 \mathrm{~nm}$ wide, $12 \mathrm{~nm}$ thick), we observe only vortex (VW) and transverse (TW) walls after initialization. Once current pulses have been injected the only wall type present in this geometry is the vortex type. In set 2, which has thicker and wider wires $(560 \mathrm{~nm} / 20 \mathrm{~nm})$, the initial walls were also of the VW and TW type, but changed into a variety of domain walls with multiple vortices after pulse injection. The same was the case for set 4 , the thickest wires under investigation $(500 \mathrm{~nm} / 34 \mathrm{~nm})$, but here only VWs were initialized. In set 3 , rather thick and wide wires $(560 \mathrm{~nm} / 30 \mathrm{~nm})$, besides VWs also walls consisting of two vortices were present after initialization and after the application of current pulses domain walls with two to four vortices were observed.

In previous studies we found [19] that, for the geometry of the thin and narrow wire, the domain wall should be a transverse wall after initialization, while in the other three geometries vortex walls are expected. However the energies of the transverse and the vortex walls are for the thinnest and narrowest geometry quite similar and for the other geometries the VW has a lower energy compared to the TW.

For the thicker wires VWs were only observed right after initialization. As soon as pulses of a certain strength $\left(0.69 \mathrm{~A} / \mathrm{m}^{2}\right.$ to $\left.2.88 \times 10^{11} \mathrm{~A} / \mathrm{m}^{2}\right)$ have been injected the VWs irreversibly transformed into more complicated spin structures. The most common type is the $2 \mathrm{AP}$ (two vortices with antiparallel chirality) type pictured in Fig. 1(a)-(c) and on subsequent injection of stronger pulses a variety of different multivortex walls was obtained, whereby the $2 \mathrm{AP}$ remained the most common one. Thus these observations and simulations suggest that in the thicker and wider wires, which are far away from the VW-TW phase boundary [19], the 2AP type (Fig. 1(a)-(c)) can be more favorable than a VW. As explained in [28] a vortex domain wall is not perfectly symmetric but consists of a topological vortex defect and two topological edge defects connected by an in-plane domain wall. Charges accumulate at these edge defects which thus serve as sources for stray fields. In the schematic sketches in Fig, 1(a), (d), (g) these edge defects are, depending on the sign of their charge, marked by either solid red or dotted blue circles and the connecting diagonal shows up as a bright or dark contrast line, depending on the chirality, as expected in Fresnel mode. In a 2AP domain wall, the two topological vortex defects are balanced by a total of four edge defects. Other spin structures which occur in sets $2-4$ after pulses have been injected are $3 \mathrm{AP}$ domain walls (Fig. 1(d)-(f)), which are similar to the 2AP walls with the difference that they consist of 


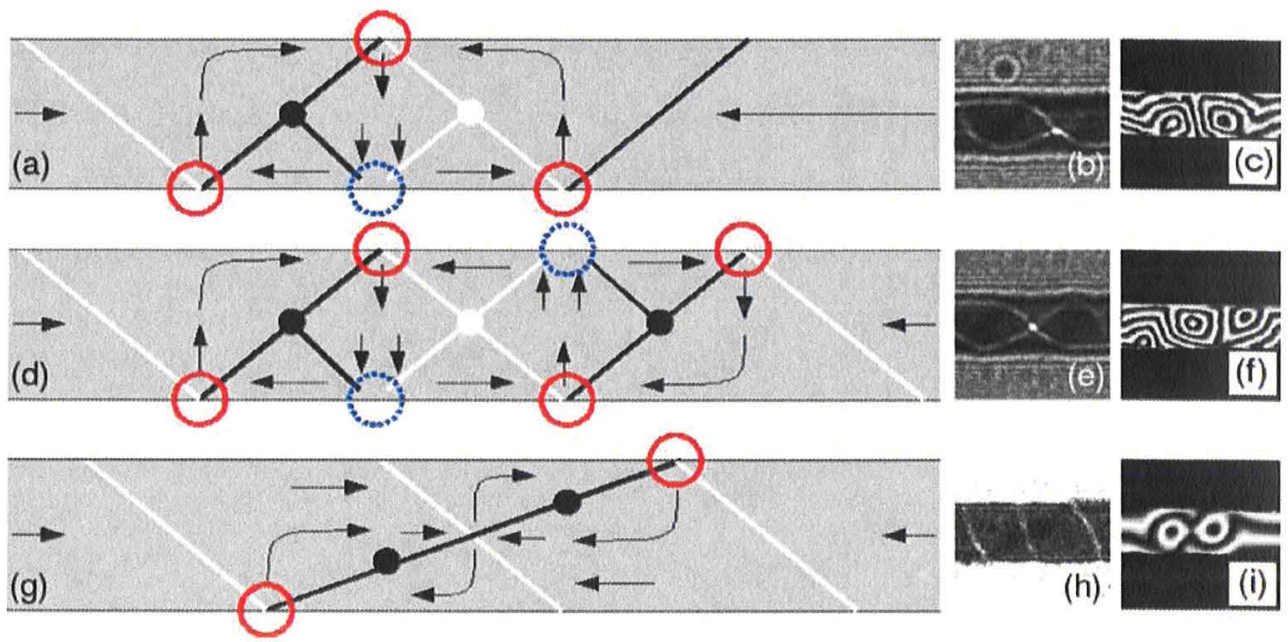

Fig. 1 (online colour at: www.pss-a.com) (a), (d), (g) Schematic spin structures, (b), (e), (h) Fresnel images and (c), (f), (i) off-axis holograms of multivortex walls taken sequentially on the same part of the sample, whereby (i) is a simulated image. The red (solid) and blue (dotted) circles indicate positions of opposite-sign magnetic charge accumulation. The black and white contrast in the schematic drawings corresponds to overfocus Fresnel images and for simplicity the vortex cores are drawn in the center of the wire. The images show (a)-(c) a $2 \mathrm{AP}$ domain wall, (d)-(f) a 3AP domain wall and (g)-(i) a 2P domain wall. The contrast appearing at the sample edge is omitted in the schematic images.

three vortices with alternating chirality, and $2 \mathrm{P}$ (two vortices with parallel chirality) domain walls (Fig. 1(g)-(i)). In the 2P spin structure an antivortex is formed between the two vortices for topological reasons. Because an antivortex has twice the winding number of an edge defect and the total winding number of a domain wall has to be zero [29] there are only two edge defects in such a structure, which are placed at opposite ends of the domain wall. Three examples of spin structures which occurred less often are domain walls consisting of three vortices with the same chirality separated by antivortices, domain walls which consist of $2 \mathrm{P}$ domain walls with an extra vortex of opposing chirality at one end, and domain walls which are $2 \mathrm{P}$ domain walls with an antiparallel rotating vortex at both sides. The fact that the positions of these extra vortices are shifted to the edge of the wire, like those of the outer vortices of the 3AP domain wall (Fig. 1(f)), is an indication that they are pinned by edge defects.

The influence of geometry on the occurrence of either a VW or TW has been discussed in Ref. [26]. The spin structures after initialization depend on the interplay of exchange and stray field energy, which scale differently with the wire dimensions. The more complicated structures offer the possibility to increase the separation between same sign stray field sources (Fig. 1(g)) or allow flux closure through the initialization of opposite sign stray field sources (Fig. 1(a), (d)), thus decreasing the stray field. But at the same time at the center of the (anti) vortices the magnetizations has to rotate out of the easy plane. In [19] it was found that in a particular geometry TWs and VWs are separated by an energy barrier of 6.7 to $8.0 \times 10^{-21} \mathrm{~J}$, the energy needed to nucleate the vortex. There are thus geometries where the TW is a metastable state. The same is true for the thicker wires in our experiments, only here the VWs are the local minima which are separated from the energetically lower lying multivortex states by energy barriers imposed by the need to nucleate extra vortices and antivortices.

Because TEM measurements require the substrate to be very thin, the heat transport in our samples is rather poor. Thus when injecting current pulses, the temperature in the wire rises significantly due to the Joule heating [20]. When monitoring the resistance of the wire in a comparable setup Togawa et al. [30] found evidence that Joule heating above the Curie temperature is possible. This large thermal energy due to the current pulses makes it possible to overcome even high energy barriers. Thus the explanation for 
the multitude of observed domain wall types is as follows: besides the initial vortex state there are several energetically lower-lying multivortex states, which are separated from each other by high energy barriers. Some of these states have the same energy for symmetry considerations, e.g. domain walls with reversed chiralities, but in general their energies vary. Due to the strong heating, transitions between the different states are possible and the energy barriers separating the different wall types lead to the fact that different spin structures can be observed at room temperature.

Besides the changes in spin structure discussed so far, it can also be energetically favorable for the domain wall to move to a different position. Within the wire, the domain walls are held in place by pinning at edge roughness or other defects. The thermal energy helps to overcome restraining forces and the domain wall can statistically hop into a different minimum. Contrary to the unidirectional motion due to spin torque, this random movement is bidirectional. The necessary current densities to move the domain walls are of the same magnitude as those needed to switch between different spin configurations. In our samples these current densities are between $0.71 \times 10^{11} \mathrm{~A} / \mathrm{m}^{2}$ for set 4 and $2.91 \times 10^{11} \mathrm{~A} / \mathrm{m}^{2}$ for set 1 . These values are lower those reported in literature for spin torque effects $[7-9,18]$, indicating that we do not observe a spin torque effect. Differences between samples with varying wire dimensions are due to the better heat dissipation for a wire with a proportionally larger interface with the substrate [20]. The current densities to overcome the barrier between the VW and 2AP domain wall is slightly lower $\left(0.69 \mathrm{~A} / \mathrm{m}^{2}\right.$ to $\left.2.88 \times 10^{11} \mathrm{~A} / \mathrm{m}^{2}\right)$ than the one needed to overcome the pinning potential and, in the thicker wires, switching occurs between different multivortex spin configurations. Sometimes, especially for moving single vortex walls, the available energy is not sufficient to fully depin the domain wall. We then observe a wall which as a whole is still pinned but the central intensity dot, marking the center of the vortex, moves back and forth. The outer parts of the wall are pinned by edge roughness, which is typically on the order of several ten nanometers (cf. Fig. 3(a)) and the vortex center is held in place by structural defects, for instance nonmagnetic holes. Therefore this yields information about the relative strenghts of these two pinning mechanisms and we find that pinning by edge roughness prevails. Another special case of thermally activated motion is a repeated jumping between two particular positions in the wire when injecting pulses. In this case the potential landscape for the domain wall is apparently a local double-well potential, whereby the separating barrier is overcome by thermal excitations due to Joule heating by the current pulses.

There were also other effects which yield information about how the potential landscape for domain walls can be changed by current pulses. For current densities similar to those for depinning, nucleation of new domains and thus also of domain walls was observed. This has been reported before $[31,32]$.

Furthermore, we found that when the domain walls within a wire are less than about $4 \mu \mathrm{m}$ apart they can interact: when a new domain wall is nucleated in the neighborhood of an existing one, its chirality will prefer to be antiparallel to the one of its neighbor. If the walls consist of more than one vortex, the core which is closest to the other wall is affected by the interaction. A similar effect was observed in the thinnest wire when two VWs came close to each other. If they have opposite chirality, they will attract

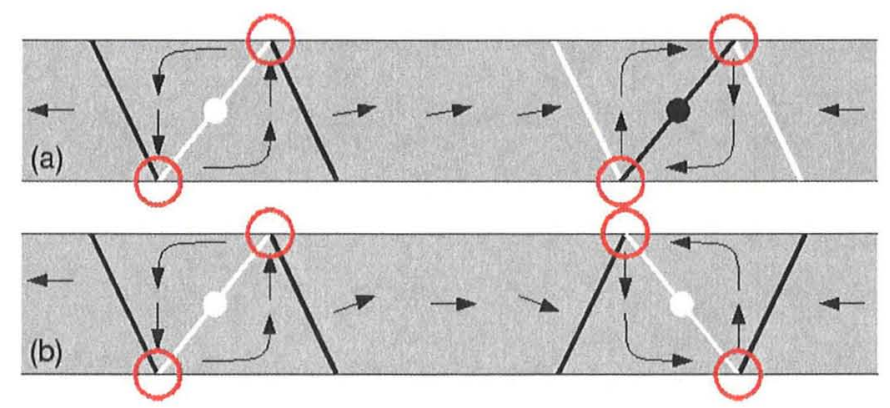

Fig. 2 (online colour at: www.pss-a.com) (a) Spins in the domain between the VWs can rotate continuously, while in (b) they face an increasing exchange energy the closer the two walls are to each other. 

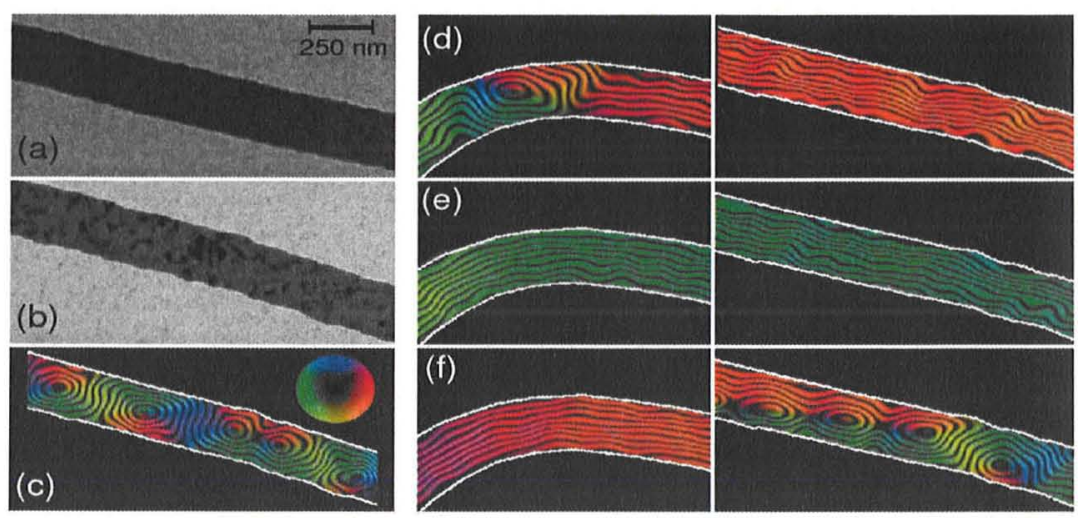

Fig. 3 (online colour at: www.pss-a.com) In-focus image of (a) an as-grown wire and (b) the wire after the structural change. (c) Magnetic induction of the region pictured in (b). (d) Holographic magnetic images of the structurally changed wire after remagnetization, (e) after one current pulse and (f) after another three pulses. The far right image in (d), (e), (f) shows the same region pictured in (b), (c).

each other once depinned by current pulses. The motion is still random but has a strong unidirectional component. The walls will move towards each other until they finally annihilate. If by contrast two walls of parallel chirality come within a certain distance of each other there is a repulsive force. Even for much higher pulses than usually needed to depin domain walls, there will be no further motion towards each other. This can be understood when taking into account the spin structure (Fig. 2). While for vortices with opposite chirality the magnetization between the two walls can continuously rotate because spins at both sides of the domain are parallel, this is not possible for VWs with the same sense of rotation. Here the magnetization cannot rotate continuously, but will face an increasing exchange energy, which will stabilize the configuration, prevent a further approach and hinder the ultimate annihilation.

There are different regimes for the changes incurred by the current injection. Reversible changes to the magnetic structure can occur as previously shown but for sufficiently high current densities, even structural changes can be induced. In sets 1 and 2 irreversible changes of the structure of one or two wires were observed. Both times this happened after pulses had been injected that were $10 \%$ or more above the current density usually needed to induce wall motion. The first observable consequence was the formation of a very long vortex chain (up to $10 \mu \mathrm{m}$, partly shown in Fig. 3(c)). It has to be pointed out that it was never the case that all four wires of a set were affected and the effect was also not confined to either single or triple kink wires. When taking in-focus images of the crystalline wire structure there was a clear difference seen before and after the pulses (Fig. 3(a), (b)). The crystallites within the wire, which appear as dark spots in Fig. 3(b), have considerably grown in size. The usual size of crystallites in Permalloy for wires like ours is between $5 \mathrm{~nm}$ and $10 \mathrm{~nm}$ [33], after the structural change they can be up to 20 times this size. The microstructural change was permanent and not reversible by remagnetization. If the wire was remagnetized, at first no differences to earlier experiments were recognizable. The initial VW was nucleated in the kink as before and the adjacent wire, where the vortex chain had been observed, was single domain until the next kink (Fig. 3(d)). However, as seen in Fig. 3(d), (e), the magnetization in the straight part of the wire is parallel to the edge but wavy as opposed to the observed smooth field lines before the change. After a single pulse with the usual depinning current density, nothing unusual is visible: the domain wall just moved out of the kink and out of the field of view (Fig. 3(e)). However, after another three pulses the wire has a similar spin structure as before remagnetizing (Fig. 3(c), (f)). The structure change was accompanied in the total sample by a rise in resistance by about $6 \%$ compared to the as-grown wire. All these observations contribute to our conclusion that the stronger current pulses must have temporarily heated the wire above the Curie temperature upon which it became 
paramagnetic. After cooling down this will lead to the formation of a multivortex state, as confirmed in simulations (not shown). In addition it seems that the strong heating has also led to a recrystallization that changes the sizes of the crystallites and perhaps leads to oxidation and/or intermixing with the $\mathrm{Au}$ capping layer, which could explain the higher resistance.

There is an additional indication that the effects presented so far are truly of thermal origin. When increasing the pulse length we find that smaller current densities are needed to trigger the various effects. Thermal effects are statistical events and as such a longer period of heating and stronger pulses, i.e. more heating, increase the probability for them to take place. Thus for longer pulses, smaller current densities are sufficient to obtain the same probability for an event to take place.

In conclusion, we have shown that (i) in sufficiently thick and wide wires the vortex wall is not the energetically most favorable spin structure and that there is a multitude of more complicated domain wall types which are usually not accessible because they are separated from the initial state by high energy barriers. Current pulses in poorly heat conducting samples can (ii) provide the necessary energy to overcome these barriers and (iii) also trigger other effects which allow us to draw conclusions on the potential landscape for the walls in the wire. We could also (iv) provide indications that the pinning of vortex walls is predominantly due to edge defects and that high current pulses can lead to structural changes accompanied by changes in the magnetization.

Acknowledgements The authors acknowledge support by the Deutsche Forschungsgemeinschaft (SFB 513), Landesstiftung Baden-Württemberg, Samsung, and the EPSRC (UK). One of the authors (E.M.H.) acknowledges support by the "Studienstiftung des deutschen Volkes" and by the DAAD, another author (D.B.) acknowledges support by the European Union (Interreg III A program), and another author (R.E.D.B.) acknowledges support by the Royal Society.

\section{References}

[1] M. Laufenberg et al., Appl. Phys. Lett. 88, 052507 (2006).

[2] M. Kläui et al., Appl. Phys. Lett. 87, 102509 (2005).

[3] M. Hayashi et al., Phys. Rev. Lett. 97, 207205 (2006).

[4] S. S. P. Parkin, U.S. Patent 6834005 (2004).

[5] D. A. Allwood et al., Science 309, 1688 (2005).

[6] L. Berger, J. Appl. Phys. 55, 1954 (1984).

[7] A. Yamaguchi et al., Phys. Rev. Lett. 92, 077205 (2004).

[8] M. Kläui et al., Phys. Rev. Lett. 95, 026601 (2005).

[9] M. Kläui et al., Phys. Rev. Lett. 94, 106601 (2005).

[10] J. C. Slonczewski, J. Magn. Magn. Mater. 159, Ll (1996).

[11] G. Tatara and H. Kohno, Phys. Rev. Lett. 92, 086601 (2004).

[12] Z. Li and S. Zhang, Phys. Rev. B 70, 024417 (2004).

[13] A. Thiaville et al., J. Appl. Phys. 95, 7049 (2004).

[14] S. Zhang and Z. Li, Phys. Rev. Lett. 93, 127204 (2004).

[15] A. Thiaville et al., Europhys. Lett. 69, 990 (2005).

[16] L. Berger, J. Appl. Phys. 63, 1663 (1988).

[17] C. Y. Hung et al., J. Appl. Phys. 67, 5941 (1990).

[18] M. Kläui et al., Appl. Phys. Lett. 88, 232507 (2006).

[19] M. Laufenberg et al., Appl. Phys. Lett. 88, 52507 (2006).

[20] C.-Y. You, I. M. Sung, and B.-K. Joe, Appl. Phys. Lett. 89, 222513 (2006).

[21] L. J. Heyderman et al., J. Magn. Magn. Mater. 290, 86 (2005).

[22] D. Backes et al., Microelectron. Eng. 83, 1726 (2006).

[23] R. E. Dunin-Borkowski, M. R. McCartney, and D. J. Smith, in: Encyclopaedia of Nanoscience and Nanotechnology, Vol. 3, edited by H. S. Nalwa (American Scientific, Stevenson Ranch, 2004), pp. 41-100.

[24] N. Chapman, Mater. Sci. Eng. B 3, 355 (1989).

[25] J. Raabe et al., J. Appl. Phys. 88, 4437 (2000).

[26] R. D. McMichael and M. J. Donahue, IEEE Trans. Magn. 33, 4167 (1997). 
[27] M. Kläui et al., Appl. Phys. Lett. 85, 5637 (2004).

[28] H. Youk et al., J. Appl. Phys. 99, 08B101 (2006).

[29] O. Tchernyshyov and G.-W. Chern, Phys. Rev. Lett. 95, 197204 (2005).

[30] Y. Togawa et al., Jpn. J. Appl. Phys. 45, 683 (2006).

[31] A. Yamaguchi et al., Appl. Phys. Lett. 86, 012511 (2005).

[32] F. Junginger et al., Appl. Phys. Lett. 90, 132506 (2007).

[33] C. A. F. Vaz et al., Phys. Rev. B 73, 054411 (2006). 